Supporting Information o1051407

\title{
Intramolecular Reactions of Hydroperoxides and Oxetanes: Stereoselective Synthesis of 1,2-Dioxolanes and 1,2-Dioxanes
}

\author{
Peng Dai and Patrick H. Dussault* \\ Department of Chemistry, University of Nebraska-Lincoln, Lincoln, NE 68588-0304 \\ pdussault1@unl.edu
}

\begin{abstract}
All reagents and solvents were used as supplied commercially, except THF and $\mathrm{CH}_{2} \mathrm{Cl}_{2}$, which were distilled from $\mathrm{Na} / \mathrm{Ph}_{2} \mathrm{CO}$ and $\mathrm{CaH}_{2}$, respectively. Unless noted, NMR spectra were acquired at $400 \mathrm{MHz}\left({ }^{1} \mathrm{H}\right)$ or $100 \mathrm{MHz}\left({ }^{13} \mathrm{C}\right)$ in $\mathrm{CDCl}_{3}$; individual peaks are reported as: multiplicity, integration, coupling constant in Hz. IR spectra were recorded as neat films on a $\mathrm{ZrSe}$ crystal with selected absorbances reported in $\mathrm{cm}^{-1}$. Reactions involving peroxides were monitored by TLC, using an $N, N^{\prime}$-dimethyl- $p$ phenylenediamine indicator; hydroperoxides yield an immediate reddish-pink spot while dialkyl or silyl/alkyl peroxides exhibit a pink or green-red color after standing or mild charring. ${ }^{1}$ Other reactions were monitored by $\mathrm{UV}$, by staining with dilute aq. $\mathrm{KMnO}_{4}$ or by staining/charring with a solution of $1 \%$ ceric sulfate $/ 2.5 \%$ ammonium molybdate in $10 \%$ aq. $\mathrm{H}_{2} \mathrm{SO}_{4}$. Mass spectroscopy was conducted at the Nebraska Center for Mass Spectrometry.
\end{abstract}

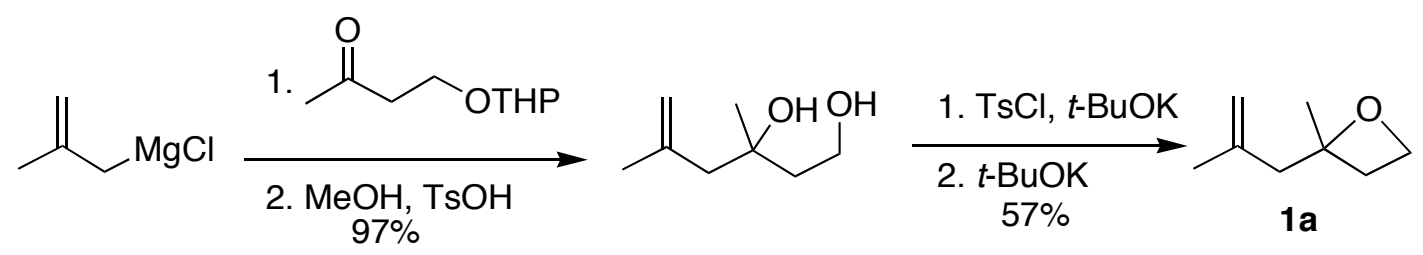

2-Methyl-2-(2-methyl-2-propenyl)-oxetane (1a): 
To a $0^{\circ} \mathrm{C}$ solution of 2-methylallyl magnesium bromide $(0.5 \mathrm{M}$ in THF, $16 \mathrm{~mL})$ was added a solution of 4-(2H-tetrahydropyran-2-yloxy)-butan-2-one ${ }^{2}$ (1.17 g, $\left.6.8 \mathrm{mmol}\right)$ in THF $(10 \mathrm{~mL})$. The reaction was stirred for $30 \mathrm{~min}$ and then quenched by addition of sat. aq. $\mathrm{NH}_{4} \mathrm{Cl}(20 \mathrm{~mL})$. The separated aqueous layer was extracted with ether $(2 \mathrm{X} 15$ $\mathrm{mL}$ ) and the combined organic layers were concentrated. The residue was dissolved in methanol $(30 \mathrm{~mL})$ and $\mathrm{TsOH}(110 \mathrm{mg})$ was added. The solution was stirred for $1 \mathrm{~h}$ and then concentrated. The residue was purified by flash chromatography (50\% EA/hex) to afford 3,5-dimethyl-5-hexene-1,3-diol (950 mg, $6.6 \mathrm{mmol}, 97 \%$, colorless oil): $\mathrm{R}_{\mathrm{f}}=0.41$ (60\% EA/hex); ${ }^{1} \mathrm{H} \delta 4.95$ (m, 1H), 4.76 (m, 1H), 3.90 (m, 2H), 2.32 (d, 1H, 13.3), 2.19 $(\mathrm{d}, 1 \mathrm{H}, 13.3), 1.85(\mathrm{~s}, 3 \mathrm{H}), 1.84(\mathrm{~m}, 1 \mathrm{H}), 1.68(\mathrm{~m}, 1 \mathrm{H}), 1.62$ (br. s, 2H), $1.27(\mathrm{~s}, 3 \mathrm{H}) ;{ }^{13} \mathrm{C}$ $\delta$ 142.4, 115.3, 73.4, 59.8, 50.2, 42.3, 27.1, 25.1; FT-IR: 3432, 2931, 1640, 1469 .

To a $0^{\circ} \mathrm{C}$ solution of the hexenediol $(375 \mathrm{mg}, 2.60 \mathrm{mmol})$ in THF $(40 \mathrm{~mL})$ was added $t$ $\mathrm{BuOK}$ (351 mg, $3.13 \mathrm{mmol}$, in one portion) followed by $p$ - TsCl (545 mg, $2.85 \mathrm{mmol}$, in $5 \mathrm{~mL}$ THF) over $30 \mathrm{~min}$. The reaction was stirred for 30 minutes at $0^{\circ} \mathrm{C}$ and then allowed to warm to room temperature. Following the disappearance of the diol ( $\sim 2 \mathrm{~h}$, TLC), the solution was re-cooled to $0^{\circ} \mathrm{C}$ and additional $t$-BuOK (450 mg, $4.0 \mathrm{mmol}$ ) was added. The reaction was stirred for 30 minutes at $0^{\circ} \mathrm{C}$ and then allowed to warm to RT. After the consumption of monotosylate (TLC, $\sim 3 \mathrm{~h}$ ) the reaction was quenched by addition of water $(50 \mathrm{~mL})$. The aqueous layer was extracted with ether $(2 \times 40 \mathrm{~mL})$. The combined organic layers were washed with brine $(100 \mathrm{~mL})$ and dried with sodium sulfate. The filtered solution was concentrated and the residue was purified by flash chromatography $(15 \% \mathrm{EA} / \mathrm{hex})$ to afford oxetane $\mathbf{1 a}(186 \mathrm{mg}, 57 \%)$ as a volatile and colorless oil: $\mathrm{R}_{\mathrm{f}}=$ 0.59 (20\% EA/hex); ${ }^{1} \mathrm{H} \delta 4.86(\mathrm{~s}, 1 \mathrm{H}), 4.72$ (s, 1H), 4.44 (m, 2H), 2.60 (m, 1H), 2.45 (AB $\mathrm{d}, 1 \mathrm{H}, 14.0), 2.38(\mathrm{AB} d, 14.0), 2.35(\mathrm{~m}, 1 \mathrm{H}), 1.79(\mathrm{~s}, 3 \mathrm{H}), 1.43(\mathrm{~s}, 3 \mathrm{H}) ;{ }^{13} \mathrm{C} \delta 142.5$, 113.8, 86.4, 64.4, 50.0, 32.8, 27.1, 23.7; FT-IR: 3068, 2937, 1453, 987, 895 


\section{2- (3,5-Dimethyl-3-(2-hydroxyethyl)-5-methoxy-1,2-dioxolane (1c)}

To a $-78^{\circ} \mathrm{C}$ solution of oxetane $1 \mathrm{a}(158 \mathrm{mg}, 1.25 \mathrm{mmol})$ in $15 \mathrm{~mL} \mathrm{CH}_{2} \mathrm{Cl}_{2}$ and $5 \mathrm{~mL}$ methanol was bubbled $\mathrm{O}_{3} / \mathrm{O}_{2}$ until the solution turned pale blue. Residual ozone was then discharged by bubbling with $\mathrm{O}_{2}$ and the reaction was warmed to RT. The residue obtained upon concentration was purified by flash chromatography on silica gel pretreated with $2.5 \% \mathrm{NEt}_{3}$ to afford a 1:1 mixture of diastereomeric dioxolanes 1c $(125 \mathrm{mg}$, $57 \%)$ as a colorless oil: $\mathrm{R}_{\mathrm{f}}=0.38(60 \% \mathrm{EA} / \mathrm{hex}) ;{ }^{1} \mathrm{H} \delta 3.74-3.85(2 \mathrm{H}), 3.31(\mathrm{~s}, 1.5 \mathrm{H})$, $3.30(\mathrm{~s}, 1.5 \mathrm{H}), 2.84$ (d, 0.5H, 12.9), 2.72 (app. t, 0.5H, 6.4), 2.53 (d, 0.5H, 12.7), 2.46 (d, 0.5H, 12.7), 2.28 (d, 0.5H, 12.9), $2.06(\mathrm{~m}, 0.5 \mathrm{H}), 1.90$ (br. s, $1 \mathrm{H}, \mathrm{OH}), 1.76(\mathrm{~m}, 0.5 \mathrm{H})$, $1.478(\mathrm{~s}, 1.5 \mathrm{H}), 1.475(\mathrm{~s}, 1.5 \mathrm{H}), 1.40(\mathrm{~s}, 1.5 \mathrm{H}), 1.38(\mathrm{~s}, 1.5 \mathrm{H}) ;{ }^{13} \mathrm{C} \delta 108.5,108.3,85.8$, 85.7, 59.3, 58.9, 58.3, 56.4, 49.3, 49.1, 41.8, 39.5, 27.9, 22.6, 18.5, 18.1; FT-IR: 3382, 2955, 1451, 1070; HRMS cald for $\mathrm{C}_{8} \mathrm{H}_{17} \mathrm{O}_{4}(\mathrm{M}+\mathrm{H})^{+}:$177.1127; Found: 177.1122.

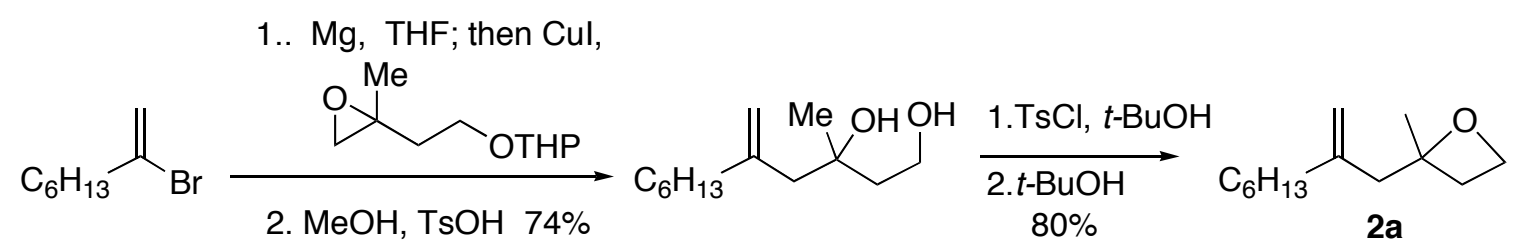

\section{2-Methyl-2- (2-methylene-octyl)-oxetane (2a):}

Into a flame-dried three-neck RBF with condenser and a dropping funnel under $\mathrm{N}_{2}$ was added THF (40 mL), magnesium turnings (500 $\left.\mathrm{mg}, 88 \mathrm{mmol}\right)$ and a tiny crystal of $\mathrm{I}_{2}$. A solution of 2-bromooctene $\mathrm{s}^{3}(2.6 \mathrm{~g}, 13.6 \mathrm{mmol})$ in THF $(10 \mathrm{~mL})$ was added dropwise while reaction was initiated by heating to approximately $40{ }^{\circ} \mathrm{C}$. After addition of bromooctene was complete, the reaction was refluxed for 3 hours. The mixture was then cooled to $0^{\circ} \mathrm{C}$ and transferred via cannula into a $0^{\circ} \mathrm{C}$ suspension of $\mathrm{CuI}(760 \mathrm{mg}, 4$ $\mathrm{mmol})$ in THF $(5 \mathrm{~mL})$. After the reaction had been stirred at $0^{\circ} \mathrm{C}$ for $\sim 30 \mathrm{~min}, 2-[2-(2-$ methyl-oxiranyl)-ethoxy]-tetrahydropyran ${ }^{4}(1.80 \mathrm{~g}, 9.7 \mathrm{mmol}$ was added as a solution in THF (10 mL). The reaction was allowed to warm to RT overnight and then quenched by 
the addition of $10 \%$ aq. $\mathrm{H}_{2} \mathrm{SO}_{4}(20 \mathrm{~mL})$ and ice $(20 \mathrm{~g})$. The aqueous layer was extracted with ether $(3 \times 30 \mathrm{~mL})$ and the combined organic layers were dried over $\mathrm{Na}_{2} \mathrm{SO}_{4}$. The residue obtained upon concentration was dissolved in $25 \mathrm{~mL}$ methanol and $\mathrm{TsOH}$ (150 $\mathrm{mg}, 1 \mathrm{mmol}$ ) was added. The solution was stirred for $30 \mathrm{~min}$ before concentrated under vacuum. The resulting residue was purified by flash chromatography (50\% EA/hex) to afford 3-methyl-5-methylene-undecane-1,3-diol (1.54 g, $7.2 \mathrm{mmol}, 74 \%$, colorless oil): $\mathrm{R}_{\mathrm{f}}=0.13(20 \% \mathrm{EA} / \mathrm{hex}) ;{ }^{1} \mathrm{H} \delta 4.92(\mathrm{~s}, 1 \mathrm{H}), 4.78(\mathrm{~s}, 1 \mathrm{H}), 3.89(\mathrm{~m}, 1 \mathrm{H}), 3.83(\mathrm{~m}, 1 \mathrm{H}), 2.83$ (s, 2H), 2.29 (d, 1H, 13.4), $2.17(\mathrm{~d}, 1 \mathrm{H}, 13.4), 2.09$ (t, 2H, 7.7), $1.80(\mathrm{~m}, 1 \mathrm{H}), 1.66(\mathrm{~m}$, $1 \mathrm{H}), 1.42(\mathrm{~m}, 2 \mathrm{H}), 1.22-1.32(6 \mathrm{H}), 1.23(\mathrm{~s}, 3 \mathrm{H}), 0.87(\mathrm{t}, 3 \mathrm{H}, 7.1) ;{ }^{13} \mathrm{C} \delta 146.6,113.7$, 73.4, 59.7, 48.3, 42.3, 37.9, 31.7, 29.0, 28.0, 27.0, 22.6, 14.0; FT-IR: 3431, 2929, 1644, $1465,1052,895$.

Conversion of the undecane diol (1.48 g, $6.91 \mathrm{mmol})$ to oxetane $2 \mathrm{a}(1.08 \mathrm{~g}, 5.51$ mmol, 80\%, colorless oil) was achieved by a similar procedure as for $\mathbf{1 a}: \mathrm{R}_{\mathrm{f}}=0.65(20 \%$ EA/hex); ${ }^{1} \mathrm{H} \delta 4.84$ (s, 1H), 4.73 (s, 1H), 4.48 (m, 1H), 4.40 (m. 1H), 2.58 (m, 1H), 2.39 (q, 2H, 13), $2.33(\mathrm{~m}, 1 \mathrm{H}), 2.06(\mathrm{t}, 2 \mathrm{H}, 7.7), 1.44(\mathrm{~m}, 2 \mathrm{H}), 1.42(\mathrm{~s}, 3 \mathrm{H}), 1.24-1.32(6 \mathrm{H})$, $0.88(\mathrm{t}, 3 \mathrm{H}, 6.7) ;{ }^{13} \mathrm{C} \delta 146.6,112.3,86.5,64.3,48.3,36.9,32.8,31.8,29.0,27.8,27.3$, 22.6, 14.0; FT-IR: 3070, 2929, 1643, 1457, 1230, 979, 893; HRMS cald for $\mathrm{C}_{13} \mathrm{H}_{25} \mathrm{O}$ $(\mathrm{M}+\mathrm{H})^{+}:$197.1905, Found: 197.1901.

1.. Mg, THF; then Cul,

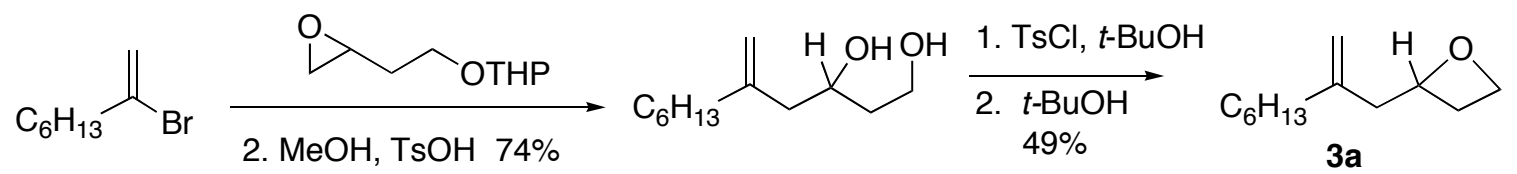

\section{2-(2-Methylene-octyl)-oxetane (3a):}

5-Methylene-undecane-1,3-diol (0.91 g, $4.6 \mathrm{mmol}, 48 \%$, colorless oil) was prepared from bromoocten $\mathrm{e}^{3}(2.39 \mathrm{~g}, 12.5 \mathrm{mmol})$ and 2-(2-oxiranyl-ethoxy)-2Htetrahydropyran $^{5}(1.48 \mathrm{~g}, 9.5 \mathrm{mmol})$ by similar procedure as described for $\mathbf{2 a}: \mathrm{R}_{\mathrm{f}}=0.18$ 
(40\% EA/hex); ${ }^{1} \mathrm{H} \delta 4.89$ (s, 1H), 4.83 (s, 1H), 3.96 (m, 1H), 3.85 (m. 2H), $2.41(\mathrm{~s}, 2 \mathrm{H})$, $2.19(\mathrm{~m}, 2 \mathrm{H}), 2.02(\mathrm{t}, 2 \mathrm{H}, 8.0), 1.72(\mathrm{~m}, 2 \mathrm{H}), 1.42(\mathrm{~m}, 2 \mathrm{H}), 1.23-1.32(6 \mathrm{H}), 0.88(\mathrm{t}, 3 \mathrm{H}$, $6.7) ;{ }^{13} \mathrm{C} \delta 146.3,112.5,69.2,61.7,44.7,38.2,35.8,31.7,29.0,27.7,22.6,14.1$; FT-IR: $3404,2928,2856,1645,1467,1056,894$; HRMS cald for $\mathrm{C}_{12} \mathrm{H}_{25} \mathrm{O}_{2}(\mathrm{M}+\mathrm{H})^{+}: 201.1855$, Found: 201.1853 .

Oxetane 3a (410 mg, $2.23 \mathrm{mmol}, 49 \%$, colorless oil) was prepared from the 1,3diol (900 mg, $4.5 \mathrm{mmol})$ by a similar procedure as for $2 \mathrm{a}: \mathrm{R}_{\mathrm{f}}=0.61(20 \% \mathrm{EA} / \mathrm{hex}) ;{ }^{1} \mathrm{H} \delta$ $4.98(\mathrm{~m}, 1 \mathrm{H}), 4.78(\mathrm{~s}, 1 \mathrm{H}), 4.70(\mathrm{~s}, 1 \mathrm{H}), 4.66(\mathrm{dt}, 1 \mathrm{H}, 8.0,5.9), 4.51(\mathrm{dt}, 1 \mathrm{H}, 9.1,5.8)$, $2.68(\mathrm{~m}, 1 \mathrm{H}), 2.53(\mathrm{dd}, 1 \mathrm{H}, 14.6,6.5), 2.38(\mathrm{~m}, 2 \mathrm{H}), 1.99(\mathrm{t}, 2 \mathrm{H}, 7.6), 1.42(\mathrm{~m}, 2 \mathrm{H}), 1.24-$ $1.32(6 \mathrm{H}), 0.88(\mathrm{t}, 3 \mathrm{H}, 6.8) ;{ }^{13} \mathrm{C} \delta 145.4,110.8,81.1,68.1,44.3,36.6,31.7,29.0,27.9$, 27.6, 22.6, 14.1; FT-IR: 3076, 2932, 1644, 1455, 1377, 1227, 977, 890; HRMS cald for $\mathrm{C}_{12} \mathrm{H}_{23} \mathrm{O}(\mathrm{M}+\mathrm{H})^{+}: 183.1749$, Found: 183.1748 .

1. Mg, THF, then Cul,

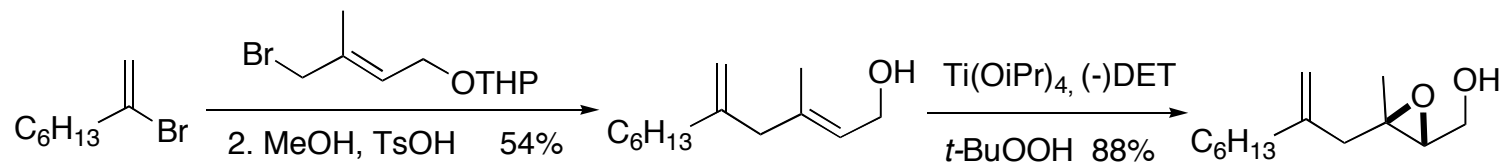

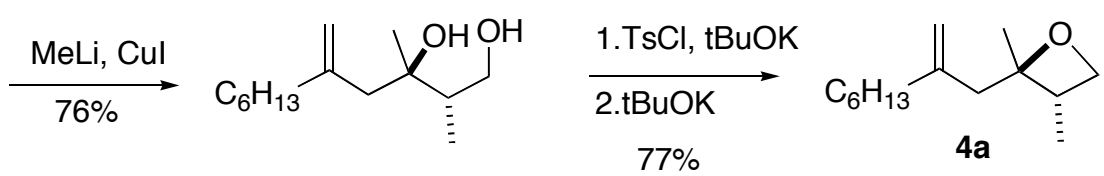

(2R,3S)-2,3-Dimethyl-2- (2-methylene-octyl)-oxetane (4a):

By a similar procedure as described above, the Grignard reagent derived from 2bromooctene $^{[2]}$ (2.27 g, $\left.11.9 \mathrm{mmol}\right)$ underwent CuI-catalyzed coupling with 2[[2(E)-4bromo-3-methyl-2-butenyl]oxy]-2H-tetrahydropyran ${ }^{6}(1.89 \mathrm{~g}, 7.6 \mathrm{mmol})$ followed by treatment with acidic methanol, to afford, after flash chromatography (20\% EA/hex), 3methyl-5-methylene-undec-2-en-1-ol (800 mg, $4.08 \mathrm{mmol}, 54 \%$, colorless oil): $\mathrm{R}_{\mathrm{f}}=0.33$ $(20 \%$ EA/hex $) ;{ }^{1} \mathrm{H} \delta 5.46$ (t, 1H, 8.1), 4.79 (s, 1H), 4.74 (s, 1H), 4.18 (t, 2H, 6.1), 2.72 (s, 
2H), $1.94(\mathrm{t}, 2 \mathrm{H}, 7.6), 1.59(\mathrm{~s}, 3 \mathrm{H}), 1.41(\mathrm{~m}, 2 \mathrm{H}), 1.24-1.33(6 \mathrm{H}), 1.18(\mathrm{OH}), 0.88(\mathrm{t}, 3 \mathrm{H}$, $6.9) ;{ }^{13} \mathrm{C} \delta 147.3,137.6,125.5,111.0,59.5,46.9,38.3,31.8,29.0,27.6,22.6,15.8,14.1$; FT-IR: 3361, 3078, 2929, 2857, 1638, 1466, 1374, 1036, 892; HRMS cald for $\mathrm{C}_{13} \mathrm{H}_{24} \mathrm{O}$ $\left(\mathrm{M}^{+}\right)$: 196.1827, Found: 196.1825.

To a $-20^{\circ} \mathrm{C}$ solution of $\operatorname{Ti}(\mathrm{O} i \text {-Pr })_{4}(1.70 \mathrm{~g}, 6.0 \mathrm{mmol})$ in $\mathrm{CH}_{2} \mathrm{Cl}_{2}(12 \mathrm{~mL})$ was added (D)- (-)-diethyltartrate (1.45 g, $7.2 \mathrm{mmol}$ in $\left.5 \mathrm{~mL} \mathrm{CH}_{2} \mathrm{Cl}_{2}\right)$ over 30 minutes via a syringe pump. The reaction was stirred for 10 more minutes, after which a solution of 3methyl-5-methylene-undec-2-en-1-ol (800 mg, $4.02 \mathrm{mmol})$ in $\mathrm{CH}_{2} \mathrm{Cl}_{2}(5 \mathrm{~mL})$ was added over 30 min via a syringe pump. After an additional $10 \mathrm{~min}$, tert-butyl hydroperoxide (5M in nonane, $2.4 \mathrm{~mL}$, dried over $4 \AA \mathrm{MS}$ ) was added over a $30 \mathrm{~min}$ period. Following consumption of starting material ( $\sim$ h, TLC), the reaction was quenched by addition of aq. tartaric acid $(10 \%, 50 \mathrm{~mL})$ and aq. $\mathrm{Na}_{2} \mathrm{SO}_{3}(10 \%, 10 \mathrm{~mL})$, and stirred until the organic layer became clear. The aqueous layer was extracted with ether $(3 \times 50 \mathrm{~mL})$, the combined organic layers were washed with brine $(100 \mathrm{~mL})$ and dried over $\mathrm{Na}_{2} \mathrm{SO}_{4}$. The filtered solution was concentrated and purified by flash chromatography $(20 \% \mathrm{EA} / \mathrm{hex})$ to afford [(2R,3R)-3-methyl-3- (2-methylene-octyl)-oxiranyl]-methanol: (760 mg, 3.58 mmol, $88 \%$, colorless oil): $\mathrm{R}_{\mathrm{f}}=0.28(20 \% \mathrm{EA} / \mathrm{hex}) ;[\alpha]_{\mathrm{D}}=-3.7\left(\mathrm{CHCl}_{3}, \mathrm{c}=1.4\right) ;{ }^{1} \mathrm{H} \delta$ $4.84(\mathrm{~s}, 1 \mathrm{H}), 4.79(\mathrm{~s}, 1 \mathrm{H}), 3.84(\mathrm{~d}, 1 \mathrm{H}, 12.0), 3.69(\mathrm{dd}, 1 \mathrm{H}, 12.0,6.8), 3.00(\mathrm{dd}, 1 \mathrm{H}, 6.7$, 4.2), $2.34(\mathrm{~d}, 1 \mathrm{H}, 14.4), 2.18(\mathrm{OH}), 2.14(\mathrm{~d}, 1 \mathrm{H}, 14.4), 2.01(\mathrm{t}, 2 \mathrm{H}, 8.0), 1.40(\mathrm{~m}, 2 \mathrm{H})$, 1.22-1.37 (9H), 0.87 (t, 3H, 6.7); ${ }^{13} \mathrm{C} \delta 145.5,112.3,62.7,61.4,60.4,45.3,36.2,31.7$, 29.0, 27.5, 22.6, 16.5, 14.1; FT-IR: 3429, 2955, 2855, 1646, 1461, 1032, 896; HRMS cald for $\mathrm{C}_{13} \mathrm{H}_{24} \mathrm{O}_{2}\left(\mathrm{M}^{+}\right):$212.1776, Found: 212.1772 .

To a $-5^{\circ} \mathrm{C}$ suspension of $\mathrm{CuI}(2.29 \mathrm{~g}, 12 \mathrm{mmol})$ in THF $(10 \mathrm{~mL})$ was added methyl lithium (18 $\mathrm{mL}, 1.5 \mathrm{M}$ in THF) over $30 \mathrm{~min}$. The initially orange reaction mixture assumed a clear tan color as the addition progressed. A solution of [(2R,3R)-3-methyl-3(2-methylene-octyl)-oxiranyl]-methanol $(610 \mathrm{mg}, 3.0 \mathrm{mmol})$ in THF $(10 \mathrm{~mL})$ was added over $30 \mathrm{~min}$. The reaction was stirred at $-5^{\circ} \mathrm{C}$ for $1 \mathrm{~h}$ and then allowed to warm to RT. 
The reaction was stirred overnight and then quenched by slow addition of sat. aq. ammonium chloride $(30 \mathrm{~mL})$. The separated aqueous layer was extracted with ether $(2 \mathrm{x}$ $20 \mathrm{~mL}$ ). The combined organic layers were washed with brine and dried over $\mathrm{Na}_{2} \mathrm{SO}_{4}$. The filtered solution was concentrated, and the residue purified by flash chromatography (30\% EA/hex) to afford (2R,3S)-2,3-dimethyl-5-methylene-undecane-1,3-diol: (500 mg, $76 \%$, colorless oil $): \mathrm{R}_{\mathrm{f}}=0.28(20 \% \mathrm{EA} / \mathrm{hex}) ;[\alpha]_{\mathrm{D}}=-7.6\left(\mathrm{CHCl}_{3}, \mathrm{c}=2.0\right) ;{ }^{1} \mathrm{H} \delta 4.96(\mathrm{~s}$, 1H), 4.81 (s, 1H), $3.77(\mathrm{dd}, 1 \mathrm{H}, 11.2,8.6), 3.57$ (dd, 1H, 11.2, 3.1), 2.27 (d, 1H, 13.5), $2.20(\mathrm{~d}, 1 \mathrm{H}, 13.5), 2.11(\mathrm{t}, 2 \mathrm{H}, 7.5), 1.88(\mathrm{~m}, 1 \mathrm{H}), 1.43(\mathrm{~m}, 2 \mathrm{H}), 1.22-1.33(6 \mathrm{H}), 1.12(\mathrm{~s}$, $3 \mathrm{H}), 0.87(\mathrm{t}, 3 \mathrm{H}, 6.7), 0.82(\mathrm{~d}, 3 \mathrm{H}, 7.1) ;{ }^{13} \mathrm{C} \delta 146.4,114.2,76.2,66.0,47.1,42.8,38.0$, 31.8, 29.1, 28.1, 22.6, 22.1, 14.1; FT-IR: 3354, 2929, 2856, 1639, 1466, 1379, 1033, 893; HRMS cald for $\mathrm{C}_{14} \mathrm{H}_{28} \mathrm{O}_{2}\left(\mathrm{M}^{+}\right)$: 228.2089, Found: 228.2086 .

(2R,3S)-2,3-Dimethyl-2- (2-methylene-octyl)-oxetane (4a) was prepared (352 mg, 1.68 mmol, 77\%) from the undecanediol (500 mg, $2.19 \mathrm{mmol}$, colorless oil) by a similar procedure as for 1a: $\mathrm{R}_{\mathrm{f}}=0.75(20 \% \mathrm{EA} / \mathrm{hex}) ;[\alpha]_{\mathrm{D}}=+7.9^{\circ}\left(\mathrm{CHCl}_{3}, \mathrm{c}=1.4\right) ;{ }^{1} \mathrm{H} \delta 4.83(\mathrm{~s}$, 1H), $4.72(\mathrm{~s}, 1 \mathrm{H}), 4.52(\mathrm{dd}, 1 \mathrm{H}, 8.3,5.8), 4.07$ (dd, 1H, 7.1, 5.9), $2.88(\mathrm{~m}, 1 \mathrm{H}), 2.43$ (AB d, 1H, 13.9), $2.36(\mathrm{AB} \mathrm{d}, 1 \mathrm{H}, 13.9), 2.04(\mathrm{t}, 2 \mathrm{H}, 7.7), 1.44(\mathrm{~m}, 2 \mathrm{H}), 1.24-1.31(9 \mathrm{H}), 1.08$ (d, 3H, 7.1), $0.88(\mathrm{t}, 3 \mathrm{H}, 6.8) ;{ }^{13} \mathrm{C} \delta 146.4,112.3,88.7,72.7,49.5,37.1,36.9,31.8,29.1$, 27.7, 22.6, 21.0, 14.1, 14.0; FT-IR: 2960, 2927, 2857, 1642, 1463, 1378, 979, 892; HRMS cald for $\mathrm{C}_{14} \mathrm{H}_{27} \mathrm{O}(\mathrm{M}+\mathrm{H})^{+}:$211.2062, Found: 211.2059 .

\section{cis and trans-2- (5-hexyl-3-(2-hydroxyethyl)-5-methoxy-3-methyl-1,2-dioxolane (2c)}

Ozonolysis of oxetane $2 \mathbf{a}(98 \mathrm{mg}, 0.50 \mathrm{mmol})$ in $3: 1 \mathrm{CH}_{2} \mathrm{Cl}_{2} / \mathrm{methanol}$ by similar procedure as employed for synthesis of 1c afforded a 1:1 mixture of diastereomeric dioxolanes $2 \mathrm{c}$ (90 mg, 0.37mmol, 73\%, colorless oil): $\mathrm{R}_{\mathrm{f}}=0.56(60 \% \mathrm{EA} / \mathrm{hex}) ;{ }^{1} \mathrm{H} \delta 3.80$ (m, 1H), $3.76(\mathrm{~m} .1 \mathrm{H}), 3.26(\mathrm{~s}, 1.5 \mathrm{H}), 3.25(\mathrm{~s}, 1.5 \mathrm{H}), 2.73(\mathrm{~d}, 0.5 \mathrm{H}, 12.9), 2.45(\mathrm{~d}, 0.5 \mathrm{H}$, 12.7), $2.40(\mathrm{~d}, 0.5 \mathrm{H}, 12.7), 2.25(\mathrm{~d}, 0.5 \mathrm{H}, 12.9), 2.20$ (br. s, OH), $2.03(\mathrm{~m}, 1 \mathrm{H}), 1.89$ (m, 
2H), $1.74(\mathrm{~m}, 1 \mathrm{H}), 1.47(\mathrm{~m}, 2 \mathrm{H}), 1.39(\mathrm{~s}, 1.5 \mathrm{H}), 1.35(\mathrm{~s}, 1.5 \mathrm{H}), 1.22-1.32(6 \mathrm{H}), 0.88(\mathrm{t}$, $3 \mathrm{H}, 6.8) ;{ }^{13} \mathrm{C} \delta 110.5,110.4,85.4,85.3,59.3,58.9,56.4,54.3,48.8,48.6,42.0,39.5$, 31.6, 31.1, 30.6, 29.3, 28.0, 24.9, 24.8, 22.8, 22.5, 14.0; FT-IR: 3402, 2934, 1461, 1214, 1075, 1030; HRMS cald for $\mathrm{C}_{14} \mathrm{H}_{28} \mathrm{O}_{4} \mathrm{Li}(\mathrm{M}+\mathrm{Li})^{+}$: 253.1991, Found: 253.1995.

\section{c $i$ s and trans-2- (5-Hexyl-3-(2-hydroxyethyl)-5-methoxy-1,2-dioxolane (3c):} Ozonolysis of oxetane 3a (49 $\mathrm{mg}, 0.27 \mathrm{mmol})$ as described above afforded a 1:1 mixture of diastereomeric dioxolanes $3 \mathbf{c}(48 \mathrm{mg}, 0.21 \mathrm{mmol}, 77 \%)$ as a colorless oil: $\mathrm{R}_{\mathrm{f}}=0.43$ $\left(50 \%\right.$ EA/hex); ${ }^{1} \mathrm{H} \delta 4.62(\mathrm{~m}, 0.5 \mathrm{H}), 4.47$ (m, 0.5H), $3.76(\mathrm{~m}, 2 \mathrm{H}), 3.27$ (s, 1.5H), $3.26(\mathrm{~s}$, 1.5H), 2.77 (dd, 0.5H, 12.5, 7.4), $2.63(\mathrm{dd}, 0.5 \mathrm{H}, 12.6,8.0), 2.37$ (dd, 0.5H, 12.6, 7.8), $2.21(\mathrm{~d}, 0.5 \mathrm{H}, 12.5,5.5), 1.68-1.97(3 \mathrm{H}), 1.22-1.58(10 \mathrm{H}), 0.88(\mathrm{t}, 3 \mathrm{H}, 6.8) ;{ }^{13} \mathrm{C} \delta 109.9$, $108.9,79.6,78.6,60.1,59.7,50.6,49.6,49.0,48.9,37.2,34.4,31.6,31.1,30.7,29.34$, 29.32, 25.0, 24.8, 22.5, 14.0; FT-IR: 3367, 2950, 2859, 1464, 1074; HRMS cald for $\mathrm{C}_{14} \mathrm{H}_{24} \mathrm{O}_{2} \mathrm{Li}(\mathrm{M}+\mathrm{Li})^{+}:$239.1835, Found: 239.1829 .

(5RS,3S)-5-Hexyl-3-(2S-hydroxypropyl)-5-methoxy-3-methyl-1,2-dioxolane (4c): Ozonolysis of oxetane $4 \mathbf{a}(50 \mathrm{mg}, 0.25 \mathrm{mmol})$ in $3: 1 \mathrm{CH}_{2} \mathrm{Cl}_{2} /$ methanol by a similar procedure as for 1c afforded a 1:1 mixture of diastereomeric dioxolanes 4c (46 mg, 0.18 mmol, 72\%, colorless oil): $\mathrm{R}_{\mathrm{f}}=0.41(20 \% \mathrm{EA} / \mathrm{hex}) ;{ }^{1} \mathrm{H} \delta 3.68(\mathrm{~m}, 2 \mathrm{H}), 3.61(\mathrm{dd}, 0.5 \mathrm{H}$, $11.9,6.5), 3.47(\mathrm{dd}, 0.5 \mathrm{H}, 11.3,5.4), 3.26(\mathrm{~s}, 1.5 \mathrm{H}), 3.25(\mathrm{~s}, 1.5 \mathrm{H}), 2.74(\mathrm{~d}, 0.5 \mathrm{H}, 12.9)$, $2.56(\mathrm{~d}, 0.5 \mathrm{H}, 12.6), 2.28(\mathrm{~d}, 0.5 \mathrm{H}, 12.6), 2.09(\mathrm{~d}, 0.5 \mathrm{H}, 12.9), 1.85-1.99(2 \mathrm{H}), 1.38-1.52$ $(2 \mathrm{H}), 1.35(\mathrm{~s}, 1.5 \mathrm{H}), 1.33(\mathrm{~s}, 1.5 \mathrm{H}), 1.24-1.32(6 \mathrm{H}), 0.94(\mathrm{~d}, 1.5 \mathrm{H}, 7.1), 0.93(\mathrm{~d}, 1.5 \mathrm{H}$, 7.0), $0.88(\mathrm{t}, 3 \mathrm{H}, 6.8) ;{ }^{13} \mathrm{C} \delta 110.24,110.20,88.9,87.9,65.0,64.8,52.8,51.3,48.6,48.5$, $44.0,41.3,31.62,31.61,30.5,30.4,29.4,29.3,26.7,25.0,24.8,22.51,22.49,22.0,14.1$, 14.0, 13.0; FT-IR: 3426, 2935, 1459, 1374, 1319, 1212, 1075, 1033; HRMS cald for $\mathrm{C}_{14} \mathrm{H}_{28} \mathrm{O}_{4} \mathrm{Li}(\mathrm{M}+\mathrm{Li})^{+}:$267.2148, Found: 267.2144. 
2-(2-Methyl-2-triethylsilyldioxypropyl)-2-methyl-oxetane (5a): To a 1.0 M solution of oxetane 1 a $(120 \mathrm{mg}, 0.95 \mathrm{mmol})$ in $\mathrm{CH}_{2} \mathrm{Cl}_{2}$ was added $\mathrm{Co}(\mathrm{acac})_{2}(74 \mathrm{mg}, 0.28 \mathrm{mmol})$ and $\mathrm{Et}_{3} \mathrm{SiH}(0.5 \mathrm{~mL}, 3 \mathrm{mmol})$. The reaction mixture was stirred under $\mathrm{O}_{2}(1.0 \mathrm{~atm}$, balloon) until starting material had disappeared (ca. 6h). The reaction mixture was run through a plug of Celite and the concentrated residue was purified by flash chromatography (10\% EA/hex) to afford the silylperoxide 5a (179 mg, $0.74 \mathrm{mmol}, 78 \%$, colorless oil): $\mathrm{R}_{\mathrm{f}}=0.58(20 \% \mathrm{EA} / \mathrm{hex}) ; 1 \mathrm{H} \delta 4.51(\mathrm{~m}, 1 \mathrm{H}), 4.43(\mathrm{~m}, 1 \mathrm{H}), 2.64$ (ddd, 1H, 10.8, 9.1, 7.4), 2.31 (ddd, 1H, 10.8, 8.6, 6.2), 2.11 (AB d, 1H, 14.0), 2.03 (AB d, 1H, 14.0), $1.56(\mathrm{~s}, 3 \mathrm{H}), 1.25$ (s, 3H), 0.97 (t, 9H, 7.9), 0.66 (q, 6H, 7.9); ${ }^{13} \mathrm{C} \delta 86.5,82.1$, 64.5, 49.3, 34.7, 27.9, 25.5, 25.4, 6.7, 3.9; FT-IR: 2965, 2871, 1466, 1007, 970; HRMS cald for $\mathrm{C}_{14} \mathrm{H}_{31} \mathrm{OSi}(\mathrm{M}+\mathrm{H})^{+}:$243.2144, Found: 243.2140 .

2-(2-Hydroxyethyl-3,5,5-trimethyl-1,2-dioxolane (5c): To a solution of silylperoxy oxetane $5 \mathbf{a}(60 \mathrm{mg}, 0.25 \mathrm{mmol})$ in $\mathrm{CH}_{3} \mathrm{CN}(6 \mathrm{~mL})$ was added aq. $\mathrm{HF}(40 \%, 0.3 \mathrm{~mL})$. After stirring for $10 \mathrm{~min}$, the reaction was quenched by slow addition of aq. $\mathrm{NaHCO}_{3}$ (sat. $10 \mathrm{~mL})$. The aqueous layer was extracted with ether $(10 \mathrm{~mL}$ x 2$)$. The combined organic layers were dried over $\mathrm{Na}_{2} \mathrm{SO}_{4}$ and filtered. The residue obtained upon concentration was purified by flash chromatography $(30 \% \mathrm{EA} / \mathrm{hex})$ to afford 1,2dioxolane 5c (32mg, 80\%): $\mathrm{R}_{\mathrm{f}}=0.25$ (40\% EA/hex); 1H $\delta 3.79(\mathrm{~m}, 2 \mathrm{H}), 2.33(\mathrm{~d}, 1 \mathrm{H}$, 11.9), 2.19 (d, 1H, 11.9), 2.07 (br. s., OH), 2.02 (ddd, 1H, 14.6, 6.6, 5.6), 1.83 (ddd, 1H, 14.6, 6.6, 5.8), 1.38 (s, 6H), $1.36(\mathrm{~s}, 3 \mathrm{H}) ;{ }^{13} \mathrm{C} \delta 86.2,84.1,59.3,57.6,41.5,26.7,25.9$, 24.4; FT-IR: 3457, 2936, 1463, 1051; HRMS cald for $\mathrm{C}_{8} \mathrm{H}_{17} \mathrm{O}_{3}(\mathrm{M}+\mathrm{H})^{+}$: 161.1178 , Found: 161.1182 .

2-(2-Methyl-2-triethylsilyldioxyoctyl)-2-methyloxetane (6a): By similar procedure as employed for 5a, oxetane $\mathbf{2 a}(250 \mathrm{mg}, 1.28 \mathrm{mmol})$ was converted to an inseparable 1:1 mixture of diastereomeric silyl peroxides 6a (283 mg, 71\%, colorless oil): $R_{f}=0.66(20 \%$ 
EA/hex); 1H $\delta 4.49$ (m, 1H), 4.42 (m, 1H), 2.64 (m, 1H), 2.30 (m. 1H), 2.04 (m, 2H), $1.57(\mathrm{~s}, 1.5), 1.56(\mathrm{~s}, 1.5 \mathrm{H}), 1.23-1.38(10 \mathrm{H}), 1.20(\mathrm{~s}, 1.5 \mathrm{H}), 1.18(\mathrm{~s}, 1.5 \mathrm{H}), 0.97(\mathrm{t}, 9 \mathrm{H}$, 7.9), 0.88 (m, 3H), 0.65 (q, 6H, 7.9); ${ }^{13} \mathrm{C} \delta 86.7,86.6,84.4,84.3,64.52,64.46,47.5,47.2$, $38.7,38.4,34.8,31.9,29.92,29.88,28.1,28.0,23.9,22.63,22.61,22.5,22.2,14.1,6.7$, 3.9; FT-IR: 2956, 2877, 1465, 1377, 1238, 1003, 968; HRMS cald for $\mathrm{C}_{19} \mathrm{H}_{40} \mathrm{SiO}_{3} \mathrm{Li}$ $(\mathrm{M}+\mathrm{Li})^{+}:$351.2907, Found: 351.2910.

2- (5-Hexyl-2-hydroxyethyl-3,5-dimethyl-1,2-dioxolane (6c): By a similar procedure as employed for 5c, silylperoxy oxetane $\mathbf{6 a}(30 \mathrm{mg}, 0.1 \mathrm{mmol})$ was converted to a 1:1 mixture of diastereomeric 1,2-dioxolanes 6c (18mg, 82\%, colorless oil). A small portion of the diastereomers was separable by careful chromatography, with cis-6c eluting first.

The diastereomers were assigned by the relative strength of nOe enhancements at $\mathrm{H}_{4}$ and $\mathrm{H}_{4}$ ' following irradiation of the $\mathrm{C}_{3}$ and $\mathrm{C}_{5}$ methyl groups. ${ }^{7}$ For cis-6c, $\mathrm{H}_{4}$, but not $\mathrm{H}_{4}$ ', showed strong coupling with both the $\mathrm{C}_{3}$ and $\mathrm{C}_{5}$ methyl groups. For trans-6c, $\mathrm{H}_{4}$ showed strong coupling with the $\mathrm{C}_{5}$ methyl group and $\mathrm{H}_{4}$ ' showed strong coupling with the $\mathrm{C}_{3}$ methyl group.

cis-6c: $\mathrm{R}_{\mathrm{f}}=0.41(20 \% \mathrm{EA} / \mathrm{hex}) ;{ }^{1} \mathrm{H} \delta 3.79(\mathrm{~m}, 2 \mathrm{H}), 2.33(\mathrm{~d}, 1 \mathrm{H}, 12.0), 2.12(\mathrm{~d}, 1 \mathrm{H}$, 12.0), $2.02(\mathrm{~m}, 1 \mathrm{H}), 1.80(\mathrm{~m}, 1 \mathrm{H}), 1.55-1.65(3 \mathrm{H}), 1.38(\mathrm{~s}, 3 \mathrm{H}), 1.33(\mathrm{~s}, 3 \mathrm{H}), 1.24-1.32$ (8H), $0.88(\mathrm{t}, 3 \mathrm{H}, 6.9) ;{ }^{13} \mathrm{C} \delta 86.6,85.8,59.3,56.6,41.5,39.0,31.7,29.7,24.9,24.27$, 24.26, 22.6, 14.1; FT-IR: 3426, 2931, 1461, 1370, 1057, 796; HRMS cald for $\mathrm{C}_{13} \mathrm{H}_{26} \mathrm{O}_{3} \mathrm{Li}$ $(\mathrm{M}+\mathrm{Li})^{+}:$237.2042, Found: 237.2036.

trans-6c: $\mathrm{R}_{\mathrm{f}}=0.35(20 \% \mathrm{EA} / \mathrm{hex}) ;{ }^{1} \mathrm{H} \delta 3.80(\mathrm{~m}, 2 \mathrm{H}), 2.26(\mathrm{AB} \mathrm{d}, 1 \mathrm{H}, 12.0), 2.21(\mathrm{AB} \mathrm{d}$, 1H, 12.0), $2.03(\mathrm{~m}, 1 \mathrm{H}), 1.83(\mathrm{~m}, 1 \mathrm{H}), 1.68(\mathrm{~m}, 1 \mathrm{H}), 1.59$ (br. s, OH), $1.55(\mathrm{~m}, 1 \mathrm{H}), 1.36$ $(\mathrm{s}, 3 \mathrm{H}), 1.31(\mathrm{~s}, 3 \mathrm{H}), 1.24-1.32(8 \mathrm{H}), 0.88(\mathrm{t}, 3 \mathrm{H}, 6.9) ;{ }^{13} \mathrm{C} \delta 86.5,86.0,59.3,56.5,41.4$, 39.5, 31.8, 29.7, 24.7, 24.5, 23.6, 22.6, 14.1; FT-IR: 3418, 2934, 2859, 1457, 1374, 1053; HRMS cald for $\mathrm{C}_{13} \mathrm{H}_{26} \mathrm{O}_{3} \mathrm{Li}(\mathrm{M}+\mathrm{Li})^{+}$: 237.2042, Found: 237.2039. 
2-(2-Methyl-2-triethylsilyldioxyoctyl)-oxetane (7a): By a similar procedure as employed for 5a, unsaturated oxetane $7 \mathbf{a}(80 \mathrm{mg}, 0.44 \mathrm{mmol})$ was converted to an inseparable 1:1 mixture of diastereomeric silyl peroxides 7c (116 mg, 80\%, colorless oil): $\mathrm{R}_{\mathrm{f}}=0.71(20 \% \mathrm{EA} / \mathrm{hex}) ;{ }^{1} \mathrm{H} \delta 5.06(\mathrm{~m}, 1 \mathrm{H}), 4.63(\mathrm{~m}, 1 \mathrm{H}), 4.46(\mathrm{~m}, 1 \mathrm{H}), 2.67(\mathrm{~m}, 1 \mathrm{H})$, $2.44(\mathrm{~m} .1 \mathrm{H}), 2.04(\mathrm{~m}, 2 \mathrm{H}), 1.49(\mathrm{~m}, 2 \mathrm{H}), 1.22-1.32(8 \mathrm{H}), 1.14(\mathrm{~s}, 1.5 \mathrm{H}), 1.12(\mathrm{~s}, 1.5 \mathrm{H})$, 0.97 (t, 9H, 7.9), 0.88 (m, 3H), 0.65 (q, 6H, 7.9); ${ }^{13} \mathrm{C} \delta 83.4,79.9,79.8,68.2,68.1,45.4$, $45.2,38.3,36.9,31.84,31.80,29.84,29.83,29.4,29.3,23.6,23.5,22.7,22.6,21.5,14.1$, 6.8, 3.84, 3.83; FT-IR: 2958, 2877, 1460, 1377, 1238, 976, 740; HRMS cald for $\mathrm{C}_{18} \mathrm{H}_{38} \mathrm{SiO}_{3} \mathrm{Li}(\mathrm{M}+\mathrm{Li})^{+}:$337.2750, Found: 337.2747.

2-(5-Hexyl-3-(2-hydroxyethyl)-5-methyl-1,2-dioxolane (7c): By similar procedure as employed for synthesis of 5c, silylperoxy oxetane $7 \mathbf{a}(46 \mathrm{mg}, 0.14 \mathrm{mmol}$ ) was deprotected to furnish a 1:1 mixture (NMR) of diastereomeric dioxolanes 7c $(25.5 \mathrm{mg}$, $85 \%$, colorless oil). The cis- and trans- isomers were separated by flash chromatography. cis-7c: $\mathrm{R}_{\mathrm{f}}=0.18(20 \% \mathrm{EA} / \mathrm{hex}) ;{ }^{1} \mathrm{H} \delta 4.42(\mathrm{~m}, 1 \mathrm{H}), 3.75(\mathrm{~m}, 2 \mathrm{H}), 2.56(\mathrm{dd}, 1 \mathrm{H}, 11.9$, 7.6), 1.97 (dd, 1H, 11.9, 6.7), 1.74-1.95 (3H), $1.56(2 \mathrm{H}), 1.32(\mathrm{~s}, 3 \mathrm{H}), 1.26-1.31(8 \mathrm{H})$, $0.88(\mathrm{t}, 3 \mathrm{H}, 6.9) ;{ }^{13} \mathrm{C} \delta 85.6,80.0,60.3,51.0,38.9,36.3,31.7,29.7,24.5,24.4,22.6$, 14.1; FT-IR: 3370, 2933, 2856, 1457, 1376, 1055; HRMS cald for $\mathrm{C}_{12} \mathrm{H}_{24} \mathrm{O}_{3} \mathrm{Li}(\mathrm{M}+\mathrm{Li})^{+}$: 223.1885, Found: 223.1889.

trans-7c: $\mathrm{R}_{\mathrm{f}}=0.15(20 \% \mathrm{EA} / \mathrm{hex}) ;{ }^{1} \mathrm{H} \delta \delta 4.48(\mathrm{~m}, 1 \mathrm{H}), 3.75(\mathrm{~m}, 2 \mathrm{H}), 2.45(\mathrm{dd}, 1 \mathrm{H}, 11.9$, 6.4), 2.07 (dd, 1H, 11.9, 6.7), 1.75-1.94 (3H), 1.52-1.68 (2H), 1.26-1.35 (11H), 0.88 (t, $3 \mathrm{H}, 6.9) ;{ }^{13} \mathrm{C} \delta 85.6,79.6,60.3,51.2,39.3,36.5,31.7,29.7,24.8,23.8,22.6,14.1$; FT-IR: 3381, 2935, 1455, 1378, 1054; HRMS cald for $\mathrm{C}_{12} \mathrm{H}_{24} \mathrm{O}_{3} \mathrm{Li}(\mathrm{M}+\mathrm{Li})^{+}:$223.1885, Found: 223.1888 .

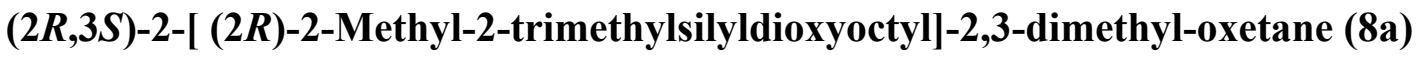

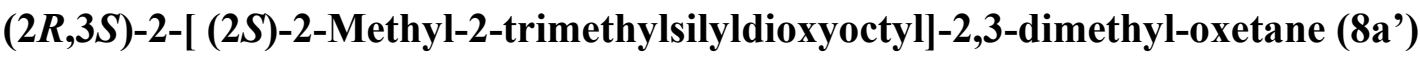


By a similar procedure as employed for $\mathbf{5 a}$, oxetane $\mathbf{4 a}(80 \mathrm{mg}, 0.38 \mathrm{mmol})$ was converted to a 1:1 mixture of diastereomeric silylperoxy oxetanes (97 mg, 71\%, colorless oil). The mixture could be carefully separated by flash chromatography to furnish nearly pure samples of diastereomers 8a and 8a':

8a: $\mathrm{R}_{\mathrm{f}}=0.63(20 \% \mathrm{EA} / \mathrm{hex}) ;{ }^{1} \mathrm{H} \delta 4.52(\mathrm{dd}, 1 \mathrm{H}, 8.4,5.9), 4.09(\mathrm{dd}, 1 \mathrm{H}, 7.5,5.9), 2.90(\mathrm{~m}$, 1H), $2.08(\mathrm{~d}, 1 \mathrm{H}, 14.9), 1.98(\mathrm{~d}, 1 \mathrm{H}, 14.9), 1.51-1.60(2 \mathrm{H}), 1.41(\mathrm{~s}, 3 \mathrm{H}), 1.23-1.32(8 \mathrm{H})$, $1.17(\mathrm{~s}, 3 \mathrm{H}), 1.08(\mathrm{~d}, 3 \mathrm{H}, 7.0), 0.97(\mathrm{t}, 9 \mathrm{H}, 7.9), 0.88(\mathrm{t}, 3 \mathrm{H}, 6.8), 0.65(\mathrm{q}, 6 \mathrm{H}, 7.9) ;{ }^{13} \mathrm{C} \delta$ $88.9,84.2,72.6,48.8,38.6,38.3,31.9,29.9,23.9,22.6,22.3,21.8,14.1,13.8,6.8,3.9$; FT-IR: 2955, 2876, 1464, 1379, 983, 805, 742; HRMS cald for $\mathrm{C}_{20} \mathrm{H}_{42} \mathrm{SiO}_{3} \mathrm{Li}(\mathrm{M}+\mathrm{Li})^{+}$: 365.3063, Found: 365.3059.

8a': $\mathrm{R}_{\mathrm{f}}=0.60(20 \% \mathrm{EA} / \mathrm{hex}) ;{ }^{1} \mathrm{H} \delta 4.51(\mathrm{dd}, 1 \mathrm{H}, 8.4,5.9), 4.08$ (dd, 1H, 7.5, 5.9), 2.91 (m, 1H), 2.02 (app. d, 2H, 5.0), 1.49-1.59 (2H), $1.43(\mathrm{~s}, 3 \mathrm{H}), 1.23-1.32(8 \mathrm{H}), 1.20(\mathrm{~s}, 3 \mathrm{H})$, $1.06(\mathrm{~d}, 3 \mathrm{H}, 7.0), 0.97$ (t, 9H, 7.9), 0.88 (t, 3H, 6.8), 0.66 (q, 6H, 7.9); ${ }^{13} \mathrm{C} \delta 88.9,84.4$, 72.6, 48.8, 38.7, 38.4, 31.9, 29.9, 23.8, 22.62, 22.55, 21.6, 14.1, 13.7, 6.7, 3.9; FT-IR: 2953, 2877, 1464, 1378, 982, 803, 742; HRMS cald for $\mathrm{C}_{20} \mathrm{H}_{42} \mathrm{SiO}_{3} \mathrm{Li}(\mathrm{M}+\mathrm{Li})^{+}: 365.3063$, Found: 365.3066.

\section{(2S)-2-[ (3S,5R)-5-Hexyl-3,5-dimethyl-1,2-dioxolan-3-yl]-propanol (8c):}

By similar procedure as employed for synthesis of $\mathbf{5 c}$, diastereomeric pure silylperoxy oxetane 8a (29 mg, $0.081 \mathrm{mmol})$ was converted to diastereomeric pure dioxolane 8c (15.6 mg, 79\%, colorless oil): $\mathrm{R}_{\mathrm{f}}=0.14(20 \% \mathrm{EA} / \mathrm{hex}) ;{ }^{1} \mathrm{H} \delta 3.73(\mathrm{~m}, 1 \mathrm{H}), 3.46(\mathrm{~m}, 1 \mathrm{H})$, $2.31(\mathrm{~d}, 1 \mathrm{H}, 12.4), 2.19(\mathrm{OH}), 2.08(\mathrm{~d}, 1 \mathrm{H}, 12.4), 2.04(\mathrm{~m}, 1 \mathrm{H}), 1.55-1.68(2 \mathrm{H}), 1.32(\mathrm{~s}$, $3 \mathrm{H}), 1.31(\mathrm{~s}, 3 \mathrm{H}), 1.26-1.31(8 \mathrm{H}), 0.93(\mathrm{~d}, 3 \mathrm{H}, 7.1), 0.89(\mathrm{t}, 3 \mathrm{H}, 7.0) ;{ }^{13} \mathrm{C} \delta 89.0,86.3$, 65.2, 52.8, 43.2, 39.1, 31.7, 29.7, 24.8, 24.1, 23.7, 22.6, 14.1, 13.3; FT-IR: 3493, 2931, 1457, 1375, 1034 . HRMS cald for $\mathrm{C}_{14} \mathrm{H}_{29} \mathrm{O}_{3}(\mathrm{M}+\mathrm{H})^{+}$: 245.2117, Found: 245.2115. 
(2S)-2-[(3S,5S)-5-hexyl-3,5-dimethyl-1,2-dioxolan-3-yl]-propanol (8c'): Silyl peroxy oxetane 8a' (28 mg, $0.078 \mathrm{mmol}$ ) was converted to diastereomeric pure dioxolane 8c' (15.5 mg, $0.064 \mathrm{mmol}, 81 \%$, colorless oil: $\mathrm{R}_{\mathrm{f}}=0.20$ (20\% EA/hex); ${ }^{1} \mathrm{H}$ NMR $\delta 3.71$ (dd, $1 \mathrm{H}, 11.3,7.0), 3.45(\mathrm{dd}, 1 \mathrm{H}, 11.3,5.4), 2.41$ (d, $J=12.2), 2.02(\mathrm{~m}, 1 \mathrm{H}), 1.99(\mathrm{~d}, 1 \mathrm{H}$, 12.2), 1.50-1.66 (2H), 1.27-1.35 (15H), 0.93 (d, 3H, 7.0), $0.88(\mathrm{t}, 3 \mathrm{H}, J=6.9) ;{ }^{13} \mathrm{C} \mathrm{NMR}$ $\delta 88.8,86.3,65.2,53.1,43.4,39.4,31.7,29.7,24.8,23.6,23.4,22.6,14.1,13.3$; FT-IR 3422, 2930, 1457, 1374, 1034; HRMS cald for $\mathrm{C}_{14} \mathrm{H}_{29} \mathrm{O}_{3}(\mathrm{M}+\mathrm{H})^{+}$: 245.2117; Found: 245.2118 .

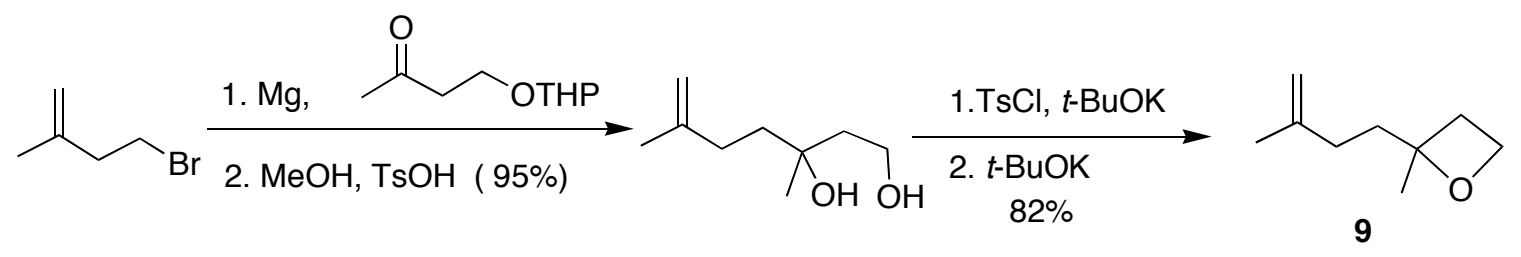

2-Methyl-2- (3-methyl-but-3-enyl)-oxetane (9):

By a similar procedure as described for the synthesis of the precursor of $\mathbf{1 a}, 3,6$ dimethyl-hept-6-ene-1,3-diol (1.61 g, $10.2 \mathrm{mmol}, 95 \%$, colorless oil) was prepared from 4-bromo-2-methyl-but-1-ene ${ }^{8}$ and 4-(tetrahydropyran-2-yloxy)-butan-2-one ${ }^{2}$ (1.84 g, 10.7 mmol): $\mathrm{R}_{\mathrm{f}}=0.16(40 \% \mathrm{EA} / \mathrm{hex}) ;{ }^{1} \mathrm{H} \delta 4.72$ (d, 2H, 0.6), 3.89 (m, 2H), 2.85 (br, s, 2H), $2.06(\mathrm{~m}, 2 \mathrm{H}), 1.82(\mathrm{~m}, 1 \mathrm{H}), 1.75(\mathrm{~s}, 3 \mathrm{H}), 1.63-1.72(3 \mathrm{H}), 1.26(\mathrm{~s}, 3 \mathrm{H}) ;{ }^{13} \mathrm{C} \delta 146.0,109.9$, 73.9, 59.9, 41.5, 40.4, 32.1, 26.6, 22.6; FT-IR: 3456, 2932, 1646, 1463, 1048 .

The diol was converted to oxetane 9 ( $85 \mathrm{mg}, 82 \%$, colorless oil) using a similar procedure as for synthesis of $1 \mathbf{a}: \mathrm{R}_{\mathrm{f}}=0.56(20 \% \mathrm{EA} / \mathrm{hex}) ;{ }^{1} \mathrm{H} \delta 4.71(\mathrm{~d}, 2 \mathrm{H}, 0.9), 4.49$ (dt, 1H, 8.8, 6.4), $4.43(\mathrm{dt}, 1 \mathrm{H}, 6.5,9.0), 2.47(\mathrm{~m}, 1 \mathrm{H}), 2.33(\mathrm{~m}, 1 \mathrm{H}), 2.07$ (t, 2H, 8.0), $1.82(\mathrm{~m}, 2 \mathrm{H}), 1.75(\mathrm{~s}, 3 \mathrm{H}), 1.42(\mathrm{~s}, 3 \mathrm{H}) ;{ }^{13} \mathrm{C} \delta 145.7,109.6,86.4,64.2,40.2,32.5,31.6$, 27.2, 22.6; FT-IR: 2970, 2876, 1446, 1374, 965, 885; HRMS cald for $\mathrm{C}_{9} \mathrm{H}_{15}\left(\mathrm{M}+\mathrm{H}-\mathrm{H}_{2} \mathrm{O}\right)^{+}$: 123.1174, Found: 123.1179.

2- (3-Hydroperoxy-3-methoxybutyl)-2-methyl-oxetane (10a): Ozonolysis of oxetane 9 (97 $\mathrm{mg}, 0.69 \mathrm{mmol}$ ) by a similar procedure as employed for 1c afforded 
hydroperoxyketal $\mathbf{1 0 a}(105 \mathrm{mg}, 0.55 \mathrm{mmol}, 80 \%)$ as a colorless oil: $\mathrm{R}_{\mathrm{f}}=0.38(50 \%$ EA/hex); ${ }^{1} \mathrm{H} \delta 9.47$ (s, 0.5H), $9.11(\mathrm{~s}, 0.5 \mathrm{H}), 4.45-4.55(2 \mathrm{H}), 3.33$ (s, $\left.1.5 \mathrm{H}\right), 3.32$ (s, 1.5H), 2.27-2.57 (2H), 1.65-2.02 (4H), $1.44(\mathrm{~s}, 1.5 \mathrm{H}), 1.41(\mathrm{~s}, 1.5 \mathrm{H}), 1.30(\mathrm{~s}, 1.5 \mathrm{H}), 1.29$ $(\mathrm{s}, 1.5 \mathrm{H}) ;{ }^{13} \mathrm{C} \delta 106.8,87.4,87.3,64.5,64.4,49.0,35.52,35.48,32.81,31.3,29.2$ 28.9, 27.8, 26.8, 18.6, 18.5; FT-IR: 3292, 2964, 1449, 1376, 1282, 1247, 1287, 1078, 997, 953, 842; HRMS cald for $\mathrm{C}_{9} \mathrm{H}_{17} \mathrm{O}_{3}\left(\mathrm{M}+\mathrm{H}-\mathrm{H}_{2} \mathrm{O}\right)^{+}$: 173.1178 , Found: 173.1172 .

\section{3,6-Dimethyl-3-(2-hydroxyethyl)-6-methoxy-1,2-dioxane (10c) and}

\section{5-methoxy-2,5-dimethyl-oxepan-2-yl-hydroperoxide (11):}

To a $0^{\circ} \mathrm{C}$ solution of oxetane $10 \mathrm{a}(90 \mathrm{mg}, 0.47 \mathrm{mmol})$ in ether $(10 \mathrm{~mL})$ was added $\mathrm{Yb}(\mathrm{OTf})_{3}\left(30 \mathrm{mg}, 0.05 \mathrm{mmol}, 0.1\right.$ eq.). The reaction was stirred at $0^{\circ} \mathrm{C}$ for $30 \mathrm{~min}$ and then warmed to RT. Following disappearance of oxetane 10a (TLC, $30 \mathrm{~min}$ ), the reaction was quenched with water. Workup and purification by flash chromatography (40\% EA/hex) afforded 11 (30 mg, 33\%) followed by 10c (10 mg, 12\%), both as colorless oils.

10c: $R_{\mathrm{f}}=0.40(50 \% \mathrm{EA} / \mathrm{hex}) ;{ }^{1} \mathrm{H} \delta 3.90(\mathrm{~m}, 1 \mathrm{H}), 3.78(\mathrm{~m}, 1 \mathrm{H}), 3.32(\mathrm{~s}, 2 \mathrm{H}), 3.31(\mathrm{~s}, 1 \mathrm{H})$, $1.73-1.89(4 \mathrm{H}), 1.64(\mathrm{~m}, 1 \mathrm{H}), 1.48(\mathrm{~m}, 1 \mathrm{H}), 1.28(\mathrm{~s}, 2 \mathrm{H}), 1.27(\mathrm{~s}, 1 \mathrm{H}), 1.18(\mathrm{~s}, 3 \mathrm{H}) ;{ }^{13} \mathrm{C} \delta$ $107.4,80.3,59.0,58.3,49.3,49.1,41.2,38.5,30.4,29.3,26.6,26.4,22.5,21.6,20.0$, 19.6; FT-IR: 3476, 2928, 1236, 1034; HRMS cald for $\mathrm{C}_{9} \mathrm{H}_{19} \mathrm{O}_{4}(\mathrm{M}+\mathrm{H})^{+}:$191.1283, Found: 191.1280 .

11: $\mathrm{R}_{\mathrm{f}}=0.53(50 \% \mathrm{EA} / \mathrm{hex}) ;{ }^{1} \mathrm{H} \delta 7.78(\mathrm{~s}, 1 \mathrm{H}), 4.01(\mathrm{t}, 1 \mathrm{H}, 12.1), 3.42(\mathrm{dt}, 1 \mathrm{H}, 12.1,4.3)$, $3.15(\mathrm{~s}, 3 \mathrm{H}), 2.08(\mathrm{~m}, 1 \mathrm{H}), 1.75-1.86(2 \mathrm{H}), 1.50-1.68(3 \mathrm{H}), 1.48(\mathrm{~s}, 3 \mathrm{H}), 1.17(\mathrm{~s}, 3 \mathrm{H}) ;{ }^{13} \mathrm{C}$ $\delta$ 108.3, 73.6, 58.1, 48.6, 41.9, 31.3, 27.6, 25.3, 23.2; FT-IR: 3347, 2943, 1580, 1375, 1282, 1159, 1133, 1104, 1073, 941, 819; HRMS cald for $\mathrm{C}_{9} \mathrm{H}_{17} \mathrm{O}_{3}\left(\mathrm{M}+\mathrm{H}-\mathrm{H}_{2} \mathrm{O}\right)^{+}$: 173.1178, Found: 173.1183. 


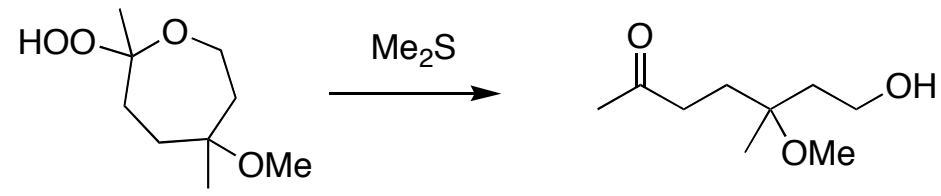

7-Hydroxy-5-methoxy-5-methyl-2-heptanone: To a solution of 11 (10 mg) in $\mathrm{CH}_{2} \mathrm{Cl}_{2}$ $(3 \mathrm{~mL})$ was added $\mathrm{Me}_{2} \mathrm{~S}(0.5 \mathrm{~mL})$. The reaction was stirred until starting material was no longer visible (TLC, $4 \mathrm{~h}$ ) after which the solution was concentrated to afford a nearly pure (NMR) sample of hydroxyketone (10 mg, quantitative, colorless oil): $R_{f}=0.13$ (50\% EA/hex); ${ }^{1} \mathrm{H} \delta 3.84(\mathrm{~m}, 1 \mathrm{H}), 3.72$ (m, 1H), 3.16 (s, 3H), 2.49 (m, 2H), 2.17 (s, 3H), $1.73-1.88(4 \mathrm{H}), 1.25$ (br. s, $1 \mathrm{H}), 1.21$ (s, $3 \mathrm{H}) ;{ }^{13} \mathrm{C} \delta 73.6,59.3,49.0,39.4,37.7,30.8$, 30.1, 22.2; FT-IR: 2443, 2929, 1712, 1376, 1075 .

2-(3-Methyl-3-triethylsilyldioxybutyl)-2-methyl-oxetane (12a): By similar procedure as employed for 5a, unsaturated oxetane $9(81 \mathrm{mg}, 0.58 \mathrm{mmol})$ was converted to silyl peroxide 12a (122 mg, $0.48 \mathrm{mmol}, 82 \%$, colorless oil): $\mathrm{R}_{\mathrm{f}}=0.65(20 \% \mathrm{EA} / \mathrm{hex}) ;{ }^{1} \mathrm{H} \delta$ $4.45(\mathrm{~m}, 2 \mathrm{H}), 2.45(\mathrm{~m}, 1 \mathrm{H}), 2.30(\mathrm{~m}, 1 \mathrm{H}), 1.76(\mathrm{~m}, 1 \mathrm{H}), 1.53-1.68(3 \mathrm{H}), 1.39(\mathrm{~s}, 3 \mathrm{H})$, 1.190 (s, 3H), 1.185 (s, 3H), 0.97 (t, 9H, 7.9), 0.66 (q, 6H, 7.9); ${ }^{13} \mathrm{C} \delta 86.7,81.8,64.2$, 36.2, 32.5, 32.0, 27.2, 24.4, 24.1, 6.8, 3.9; FT-IR: 2955, 2877, 1459, 1377, 1239, 1006, 966, 852, 807, 740; HRMS cald for $\mathrm{C}_{15} \mathrm{H}_{32} \mathrm{SiO}_{3} \mathrm{Li}(\mathrm{M}+\mathrm{Li})^{+}:$295.2281, Found: 295.2285.

3-(2-Hydroxyethyl)-3,6,6-trimethyl-1,2-dioxane (12c): To a solution of oxetane 12a (30 mg, $0.10 \mathrm{mmol})$ in ether $(3 \mathrm{~mL})$ was added aq. HF $(40 \%, 0.15 \mathrm{~mL})$. Within $1 \mathrm{~h}, 12 \mathrm{a}$ was converted to a free hydroperoxide (TLC) and the reaction was stirred until this intermediate had disappeared (48 h, TLC). Workup and purification by flash chromatography (30\% EA/hex) afforded $12 \mathrm{c}\left(13 \mathrm{mg}, 73 \%\right.$, colorless oil): $\mathrm{R}_{\mathrm{f}}=0.33$ (40\% EA/hex); ${ }^{1} \mathrm{H} \delta 4.02(\mathrm{~m}, 2 \mathrm{H}), 1.89(\mathrm{~m}, 1 \mathrm{H}), 1.57-1.78(5 \mathrm{H}), 1.29(\mathrm{~s}, 3 \mathrm{H}), 1.25(\mathrm{~s}, 3 \mathrm{H})$, $1.22(\mathrm{~s}, 3 \mathrm{H}) ;{ }^{13} \mathrm{C} \delta 82.0,73.9,59.8,41.4,35.4,31.2,26.6,24.3,24.1$; FT-IR: 3271,977 , 
1450, 1378, 1282, 1244, 847; HRMS cald for $\mathrm{C}_{9} \mathrm{H}_{19} \mathrm{O}_{3}(\mathrm{M}+\mathrm{H})^{+}$: 175.1334, Found:

175.1338.
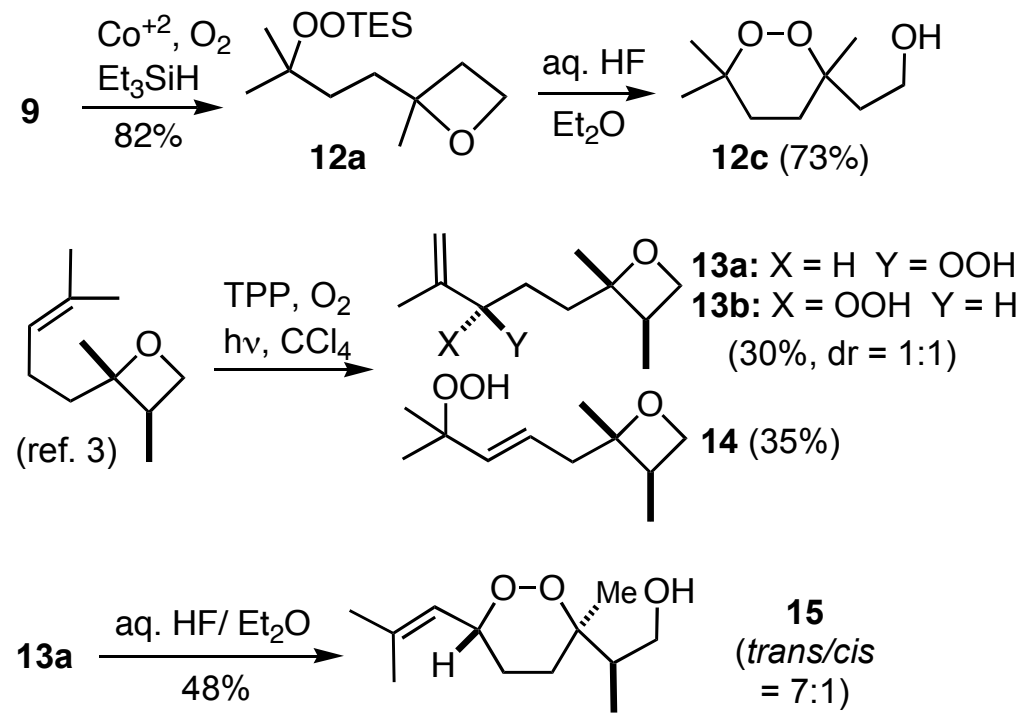

\section{5-(2S,3R-Dimethyl-oxetan-2-yl)-2-methyl-pent-1-en-3-yl-hydroperoxide}

2,3-Dimethyl-2-(4-methyl-4-pentenyl)oxetane ${ }^{9}$ (115mg, 0.68mmol) was dissolved into $10 \mathrm{~mL}$ of a $1.0 \mathrm{mM}$ solution of tetraphenylporphyrin in $\mathrm{CCl}_{4}$. The resulting solution was placed into a jacketed Pyrex cell cooled with a circulating bath $\left(-3{ }^{\circ} \mathrm{C}\right)$ into which a stream of oxygen was bubbled. The solution was irradiated with visible light $(150 \mathrm{~W}$, illuminator) for $2 \mathrm{~h}$. Two drops of a solution of BHT $\left(0.1 \mathrm{M}\right.$ in $\left.\mathrm{CH}_{2} \mathrm{Cl}_{2}\right)$ were added and the solution was concentrated. The residue was purified by flash chromatography $(40 \%$ EA/Hex) to afford $89 \mathrm{mg}(65 \%)$ of a mixture of three hydroperoxides $14, \mathbf{1 3 a}$, and $\mathbf{1 3 b}$ (approximately 2.4:1:1 ratio by NMR). Separation by semi-preparative HPLC afforded pure samples of hydroperoxyoxetanes 13a and 14:

13a: $\mathrm{R}_{\mathrm{f}}=0.38(40 \% \mathrm{EA} / \mathrm{hex}) ;{ }^{1} \mathrm{H}$ NMR $\delta 8.44(\mathrm{~s}, 1 \mathrm{H}, \mathrm{OOH}), 5.02(\mathrm{~m}, 2 \mathrm{H}), 4.55$ (dd, 1H, $J=6.0,8.2), 4.33(\mathrm{dd}, 1 \mathrm{H}, J=6.5,6.0), 4.08(\mathrm{dd}, 1 \mathrm{H}, J=6.9,6.2), 2.75(\mathrm{~m}, 1 \mathrm{H}), 1.75(\mathrm{~s}$, $3 \mathrm{H}), 1.62-1.74(4 \mathrm{H}), 1.27(\mathrm{~s}, 3 \mathrm{H}), 1.09$ (d, 3H, $J=7.1) ;{ }^{13} \mathrm{C}$ NMR $\delta$ 143.3, 114.2, 89.1, 88.7, 72.7, 38.5, 37.0, 24.2, 20.7, 17.7, 14.2; FT-IR (neat): 3379, 2979, 1459, 1377, 968, $844 \mathrm{~cm}^{-1}$; HRMS cald for $\mathrm{C}_{11} \mathrm{H}_{20} \mathrm{O}_{3} \mathrm{Li}(\mathrm{M}+\mathrm{Li})^{+}$207.1572, Found 207.1576. 
14: $\mathrm{R}_{\mathrm{f}}=0.40(40 \% \mathrm{EA} / \mathrm{hex}) ;{ }^{1} \mathrm{H}$ NMR $\delta 8.40(\mathrm{~s}, 1 \mathrm{H}, \mathrm{OOH}), 5.67(\mathrm{~m}, 2 \mathrm{H}), 4.51(\mathrm{dd}, 1 \mathrm{H}$, $J=6.0,8.3 \mathrm{~Hz}), 4.07(\mathrm{dd}, 1 \mathrm{H}, J=6.8,6.0 \mathrm{~Hz}), 2.78(\mathrm{~m}, 1 \mathrm{H}), 2.38(\mathrm{~d}, 2 \mathrm{H}, J=6.0 \mathrm{~Hz})$, $1.32(\mathrm{~s}, 3 \mathrm{H}), 1.31$ (s, 3H), 1.27 (s, 3H), 1.07 (d, 3H, $J=7.1 \mathrm{~Hz}) ;{ }^{13} \mathrm{C}$ NMR $\delta$ 137.6, 125.3, 88.6, 81.8, 72.7, 46.1, 36.2, 29.7, 24.4, 24.1, 21.2, 14.1; FT-IR (neat): 3411, 2976, 1453, 1379, $970 \mathrm{~cm}^{-1}$; HRMS cald for $\mathrm{C}_{11} \mathrm{H}_{20} \mathrm{O}_{3} \mathrm{Li}(\mathrm{M}+\mathrm{Li})^{+}$207.1572, Found 207.1574.

\section{(2R,3R,6R)-2-(6-(2-propenyl)-3-methyl-1,2-dioxan-3-yl)-propan-1-ol (15a):}

To a solution of hydroperoxyoxetane $13 \mathrm{a}(13.0 \mathrm{mg}, 0.065 \mathrm{mmol})$ in ether $(3 \mathrm{~mL})$ was added aq. HF (40\%, $0.15 \mathrm{~mL})$. The reaction was accomplished in 2 days (TLC). Workup and purification by flash chromatography (40\% EA/hex) afforded a 7:1 mixture (NMR) of diastereomeric 1,2-dioxanes $(6.2 \mathrm{mg}, 50 \%)$ from which the major isomer could be separated by analytical HPLC: $\mathrm{R}_{\mathrm{f}}=0.27$ (40\% EA/hex); ${ }^{1} \mathrm{H}$ NMR $\delta 4.97(\mathrm{~m}, 1 \mathrm{H}), 4.93$ (m, 1H), 4.39 (dd, 1H, $J=3.1,8.7), 3.80$ (ddd, 1H, $J=4.0,6.5,11.1), 3.46$ (ddd, 1H, , $J$ $=5.3,7.5,11.1), 1.82-1.95(4 \mathrm{H}), 1.77(\mathrm{~s}, 3 \mathrm{H}), 1.62-1.68(2 \mathrm{H}), 1.39(\mathrm{~s}, 3 \mathrm{H}), 0.95(\mathrm{~d}, 2 \mathrm{H}, J$ $=7.1) ;{ }^{13} \mathrm{C}$ NMR $\delta 142.7,113.1,84.0,83.3,64.4,44.5,28.9,24.3,19.7,19.4,11.8$; FTIR (neat): $3408,2969,1451,1373,1027,898 \mathrm{~cm}^{-1}$; HRMS cald for $\mathrm{C}_{11} \mathrm{H}_{20} \mathrm{O}_{3} \mathrm{Li}(\mathrm{M}+\mathrm{Li})^{+}$ 207.1572, Found 207.1569. The assignment of trans stereochemistry for the 1,2-dioxane in $15 \mathrm{a}$ was based upon the $19.7 \mathrm{ppm}{ }^{13} \mathrm{C}$ NMR chemical shift for the $\mathrm{C}_{6}$ methyl substituent and the $8.0 \mathrm{~Hz}$ coupling constant between $\mathrm{H}_{3}$ and $\mathrm{H}_{4}{ }^{10,11}$

\section{References:}

\footnotetext{
${ }^{1}$ Smith, L. L.; Hill, F. L. J. Chromatogr. 1972, 66, 101-109.

${ }^{2}$ Bellassoued, M.; Gaudemar, M. J. Organometallic Chem. 1975, 93 (1), 9-14.

${ }^{3}$ Barrett, A. G. M.; Beall, J. C.; Braddock, D. C.; Flack, K.; Gibson, V. C.; Salter, M. M. J. Org. Chem. 2000, 6508-14.

${ }^{4}$ Meyers, A. I.; Schwartzman, Steven Tetrahedron Lett. 1976, 2417-18.

${ }^{5}$ Kocienski, P.; Yeates, C. Tetrahedron Lett. 1983, 3905-6.

${ }^{6}$ Van, T. N.; Kimpe, N. D. Tetrahedron, 2000, 7969-73.

${ }^{7}$ Dussault, P. H.; Liu, X. Org. Lett., 1999, 1 (9), 1391-4.

${ }^{8}$ Leach, A. G.; Wang, R.; Wohlhieter, G. E.; Khan, S. I.; Jung, M. E.; Houk, K. N. J. Am. Chem. Soc. 2003, 4271-8.

${ }^{9}$ Dussault, P. H.; Trullinger, T. K.; Noor-e-Ain, F. Org. Lett. 2002, 4, 4591.

${ }^{10}$ Capon, R. J.; Macleod, J. Tetrahedron, 1985, 3391-3404.

${ }^{11}$ Manes, L. V.; Bakus, G. J.; Crews, P. Tetrahedron Lett., 1984, 931-4.
} 